\title{
Mitotic, but not meiotic, oriented cell divisions in rat spermatogenesis
}

\author{
Raul Lagos-Cabré and Ricardo D Moreno \\ Departamento de Ciencias Fisiológicas, Facultad de Ciencias Biológicas, Pontificia Universidad Católica de Chile, \\ Alameda 340, 8331010 Santiago, Chile \\ Correspondence should be addressed to R D Moreno; Email: rmoreno@bio.puc.cl
}

\begin{abstract}
The process of mammalian spermatogenesis involves both mitosis and meiosis at the same developmental age. Most previous studies have focused on mitotic spindle orientation during development, but not during meiotic division. Therefore, we asked whether there is a difference between mitotic and meiotic germ cell spindle orientation during rat spermatogenesis. Our results showed that mitotic spindles of spermatogonia were mainly oriented with angles ranging from 60 to $90^{\circ}$, perpendicular in relation to the basement membrane of the seminiferous tubules. On the other hand, meiotic spindles showed a random orientation. Nocodazole treatment (at a concentration that depolymerizes only astral microtubules) induced a significant increase in cells with an angle between 0 and $30^{\circ}$ (parallel) in relation to the basement membrane. Meiotic spindles did not show a significant change in their orientation after the Nocodazole treatment. Therefore, our results suggest differences between the mechanisms controlling positioning and orientation of mitotic and meiotic spindles during rat spermatogenesis. It seems that a phylogenetically conserved programme controls the mitotic spindle orientation in organisms ranging from worms to mammals.

Reproduction (2008) 135 471-478
\end{abstract}

\section{Introduction}

Organ shape depends on the coordination between cell proliferation and the spatial arrangement of cells during development (Geldmacher-Voss et al. 2003, Baena-Lopez et al. 2005, Lechler \& Fuchs 2005, Na \& Zernicka-Goetz 2006). Much is known about the mechanisms that regulate cell proliferation, but the processes by which the cells are orderly distributed remain unelucidated. Cell distribution can be accomplished either by random cell division and the later local cell migration to new positions (cell allocation) or through polarized cell division (oriented cell division; OCD; Watt \& Hogan 2000). Recent data suggest that OCD is involved in some morphogenetic processes such as vertebrate gastrulation, neurulation and retina development (Adams 1996, Tibber et al. 2004, BaenaLopez et al. 2005, Lechler \& Fuchs 2005). There are a myriad of studies on the mitotic spindle orientation during development; however, only a handful of studies have focused on meiosis, a division process unique to germ cells (Russell et al. 1990). Mammalian ovaries contain hundreds of germ cells arrested in the prophase of the first meiosis. On the contrary, mammalian spermatogenesis is characterized by constant production of spermatozoa throughout male reproductive life. Therefore, mammalian spermatogenesis seems to be the perfect model to study both mitotic and meiotic spindle behaviours in the same tissue.

Spermatogenesis is a highly ordered process that takes place in the seminiferous tubules of the mammalian testis. In adult mammals, spermatogenesis can be functionally divided into three main phases: spermatogonial proliferation (germ stem cells), meiosis of spermatocytes and differentiation of haploid spermatids (spermiogenesis; Russell et al. 1990, de Rooij \& Russell 2000). Seminiferous tubules of newborn rats contain centrally located gonocytes, which later settle on the basement membrane of the seminiferous tubules upon reaching the age of 5 days. Several morphological and flow cytometry studies have indicated that by days $6-7$, the testis contains somatic cells and spermatogonial cells, both attached to the tubule basement membrane (Billig et al. 1995, Malkov et al. 1998). In the rat, meiosis begins at days 13-14 when the first meiotic cells in the leptotene stage can be distinguished. Meiotic cells undergoing the first and second division are termed primary and secondary spermatocytes respectively. Primary spermatocytes arise from type B spermatogonia that lose contact with the basement membrane of the seminiferous tubules. As meiosis continues, a secondary spermatocyte gives rise to two haploid cells termed spermatids, which can be first observed in 24- and 25-day-old rats (Billig et al. 1995, Malkov et al. 1998). 
Thus, the first round of spermatogenesis in rats is an excellent model to sequentially study the different steps of germ cell differentiation in mammals.

Several studies in Drosophila germ cells have clearly shown that germ stem cells are attached to the basement membrane (Li \& Xie 2005, Kirilly \& Xie 2007). OCD has been observed in these cells and the mitotic spindle is perpendicularly oriented to the basement membrane. In this way, one of the daughter cells remains attached to the basement membrane as a stem cell and the other is destined for the differentiation pathway. Several studies in model organisms such as Chaetopterus or Caenorhabditis elegans oocytes have shown that OCD is a process likely driven by interactions among astral microtubules, the motor protein dynein and the cell cortex (Labbe et al. 2004, McCarthy \& Goldstein 2006). Experiments with spindle cutting and interference RNA have demonstrated that PAR (partitioning defective) and G-proteins function to generate an imbalance in pulling forces (Bellaiche \& Gotta 2005). In addition, it has been shown that atypical $\mathrm{PCK} \zeta$ (protein kinase $\mathrm{C} \zeta$ ) and CDC42 (cell division cycle 42), a small GTPase belonging to the $\rho$ family, are required for meiotic spindle migration during oogenesis in mammalian oocytes (Na \& Zernicka-Goetz 2006).

There are two previous studies suggesting that mammalian spermatogonia might not be randomly distributed along the seminiferous tubules (ChiariniGarcia et al. 2001, 2003). However, these studies did not address the question of spindle orientation during spermatogonia positioning in the seminiferous tubules. 'Spermatogonia' refers to a heterogeneous population of mitotically dividing cells resting on the basement membrane of seminiferous tubules. These cells include A-single (isolated spermatogonia, also known as $A_{s}$ ), A-paired (interconnected pairs of spermatogonia; $A_{p r}$ ) and A-aligned (chains of 4, 8, 16 or occasionally 32 spermatogonia; $\mathrm{A}_{\mathrm{al}}$ ). There are no known unique biochemicals or phenotypic markers to distinguish the cells in these spermatogonia populations and they are collectively referred to as undifferentiated spermatogonia (de Rooij \& Russell 2000). However, in the adult rat, the different subtypes can be recognized based on the stage of each cross section of the seminiferous epithelium. Spermatogonia have a long cell cycle $(\sim 70 \mathrm{~h})$ and the chance to observe mitotic spindles is minimal in the adult animal. Contrary to the adult, the histology of the prepubertal testis is quite simple and shows spermatogonia proliferating, thereby increasing the probability of observing a mitotic spindle. These reasons made us opt for the study of the behaviour of mitotic and meiotic spindles during the first round of spermatogenesis, even though it is not possible to distinguish the different subtypes of spermatogonia under these conditions. Our hypothesis is that OCD contributes to the maintenance of spermatogonia renewal during the proliferative phase of spermatogenesis and that it participates in positional signalling, committing spermatogonia to differentiation. On the other hand, it may also be possible that meiotic division does not require an oriented spindle since all daughters will be destined towards the same differentiation pathway.

Thus, the objective of this work is to determine whether there is a difference between the orientation of the spindle in germ cells undergoing mitosis (spermatogonia) and meiosis (spermatocytes) during the first wave of spermatogenesis in the rat.

\section{Results}

The average spindle angle of 5-day-old rats $\left(45.54 \pm 1.07^{\circ}\right)$ was significantly smaller than the rest of the studied ages (Fig. $1 \mathrm{~A} ; P<0.05$ ). The average spindle angle of 20-day-old rats was the largest $\left(51 \pm 1.05^{\circ}\right)$, and it was significantly larger than in 5- and 30-day-old rats. Next, we wondered whether we could detect more subtle differences in the spindle orientation of dividing germ cells in the different developmental ages. To this end, we divided the observed angles into three different groups: spindles with a parallel $\left(0-30^{\circ}\right)$, perpendicular $\left(60-90^{\circ}\right)$ or random $\left(30-60^{\circ}\right)$ orientation relative to the basement membrane of the seminiferous tubule. The proportion of spindles in the range of $60-90^{\circ}$ in 5-day-old rats was $36.78 \pm 2.09 \%$ and was significantly higher than in the other two groups of the same age (Fig. 1B). Interestingly, the proportion of spindles in the range of $60-90^{\circ}$ (perpendicular) was more than $35 \%$ in all other studied ages as well, and this number resulted to be significantly higher compared with the other groups (Fig. 1B; $P<0.05$ ).

A more in-depth analysis of the frequency of the spindle angles of all developmental ages pooled together showed a marked skew towards higher angles, with the highest frequency of cells presenting a spindle angle between 70 and $80^{\circ}$ (Fig. 2F). Analysis of the frequency of angles at each developmental age showed that there was a clear preference for angles ranging from 70 to $80^{\circ}$ in rats of 10 and 20 days of age (Fig. 2B and C). Rats of 15
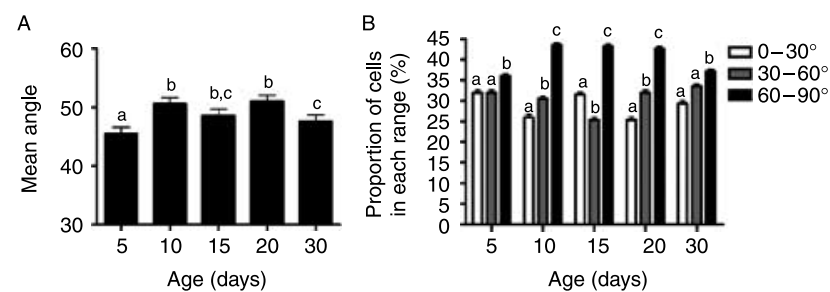

Figure 1 The orientation of the spindle in germ cells changes with age. Histological sections of three different rats of the specified ages were used to measure the spindles of dividing cells. (A) The graph represents the mean angle \pm s.D. of 600 spindles measured at each specified age. (B) The graph shows the percentage of spindles in three different ranges $\left(0-30^{\circ}, 30-60^{\circ}\right.$ and $\left.60-90^{\circ}\right)$. Different letters indicate significant differences $(P<0.05)$. 


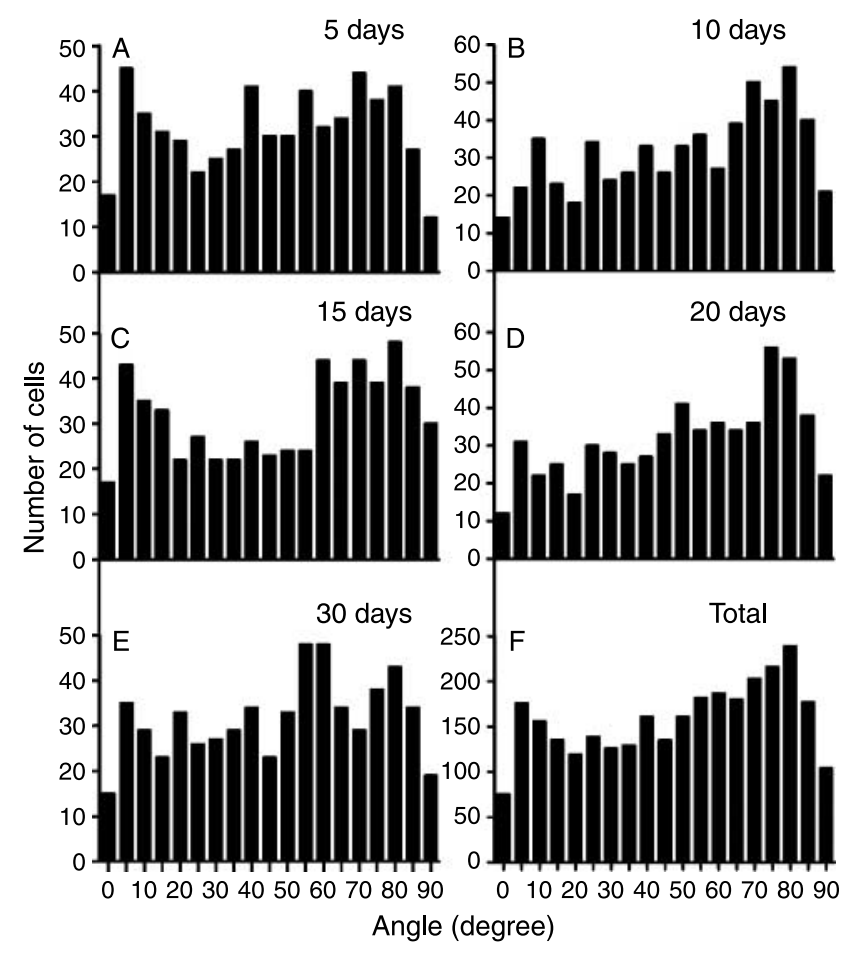

Figure 2 Distribution of spindle angles at different ages. The measured spindle angles were collected and then graphed as the frequency of each angle in rats of (A) 5, (B) 10, (C) 15, (D) 20 and (E) 30 days of age. The figure represents 600 measurements at each age. (F) The graph shows all measured angles, obtained from the different ages, collected in one data set (a total of 3000 spindles). It is clear that the highest frequency of angles has an orientation of $75-80^{\circ}$ (perpendicular) relative to the basement membrane.

and 30 days of age showed a broader range, but still with a high frequency of cells with angles $>60^{\circ}$ (Fig. 2). Thus, these results clearly indicate that germ cell spindles have a marked preference for the OCD, i.e. in a perpendicular way in relation to the basement membrane of the seminiferous tubules.

We then asked whether there was any difference between the spindle orientation in mitotic and meiotic divisions at the same age. During spermatogenesis, mitotically dividing cells are attached to the basement membrane and they are easily observed under light microscopy (Fig. 3A). On the other hand, meiotically dividing cells are always closer to the lumen of the seminiferous tubule, in the second or third cell row (Fig. 3B). Therefore, these two types of cell division can be readily identified in this tissue. As mentioned earlier, the first meiotic cells (spermatocytes) appear in 15-dayold rats; hence, we studied only rats of 15, 20 and 30 days of age for this set of experiments (Billig et al. 1995, Malkov et al. 1998). The proportion of mitotic cells with their spindle oriented in the range of $60-90^{\circ}$ was significantly higher than cells with their spindle oriented between 30 and $60^{\circ}$ or between 0 and $30^{\circ}$ (Fig. 4A). On the other hand, meiotic spindles did not show a preference as clear as mitotic spindles and they did not show any particular tendency towards large or small angles (Fig. 4B). We found that only in 20-day-old rats the meiotic spindles in the range of $0-30^{\circ}$ showed a significant difference in comparison with the other two ranges (Fig. 4B). An analysis of the frequency of the spindle angles showed that mitotic germ cells have a clear preference for perpendicular angles, with the highest number of cells presenting an $80^{\circ}$ spindle angle (Fig. 4C). Although meiotic germ cells showed a preference for angles around $55^{\circ}$ (Fig. 4D), the bars were more evenly distributed and were not skewed towards a certain angle as was the case with mitotic cells. Thus, the spindles of mitotically dividing spermatogonia present a preference for perpendicular orientation relative to the basement membrane, while this orientation was not observed in meiotic cells.

\section{Nocodazole changes the orientation of mitotic spindles}

Next, we investigated whether the OCD observed in mitotically dividing cells is dependent on the pulling forces of astral microtubules. Nocodazole is a drug, which in a low concentration $(40 \mathrm{nM})$ is able to depolymerize only astral microtubules and not those of the mitotic spindles (O'Connell \& Wang 2000). Nocodazole $(40 \mathrm{nM})$ was injected into the testes of
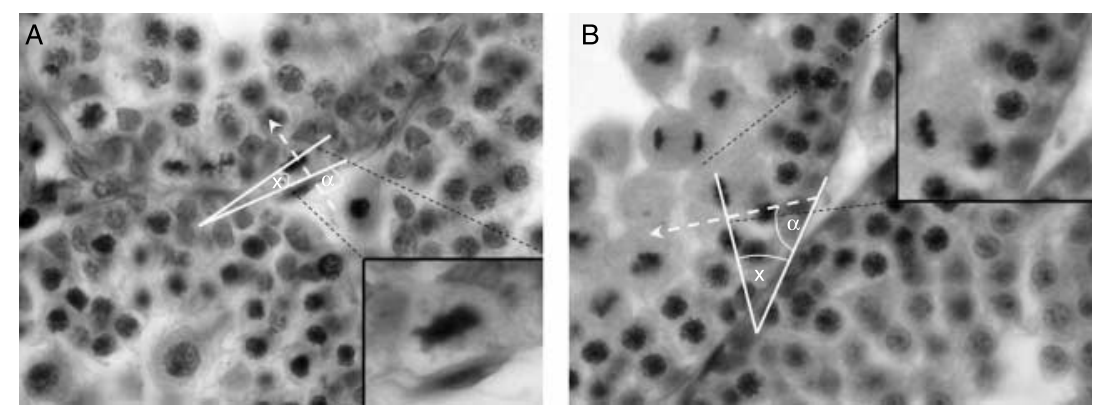

Figure 3 Determination of angles in mitotic and meiotic germ cells. The figures show how the spindle measurement was determined only in rounded tubule cross sections. (A) The metaphase plate corresponds to a dividing spermatogonium; the insert shows a magnification of the same picture. (B) A metaphase plate of a spermatocyte; the insert shows a magnification of the same cell. Solid lines indicate the angle of the metaphase plate relative to the basal membrane (angle X). The dotted lines represent the angle of the spindle (angle $\alpha$ ) that was calculated as stated in Materials and Methods. 

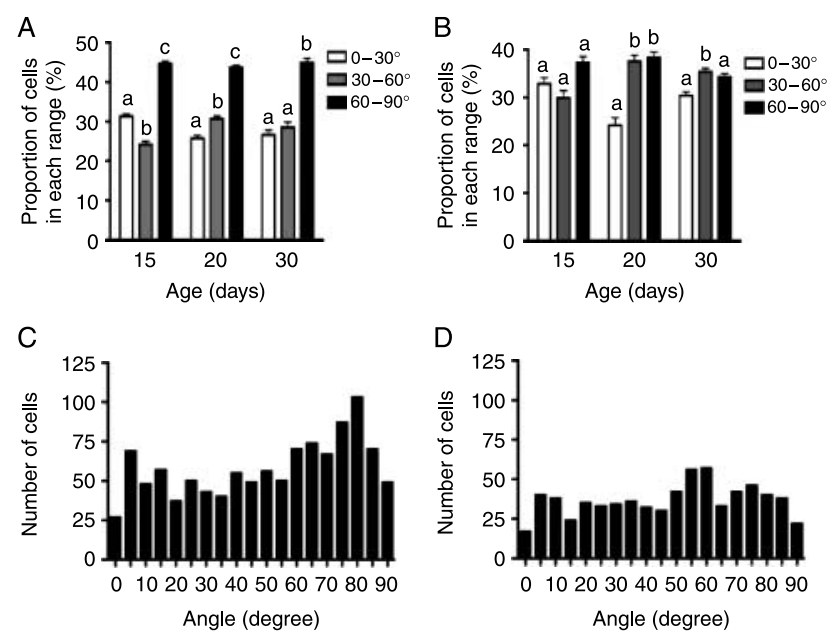

Figure 4 Mitotic and meiotic spindles have different orientations during the same developmental stage. Spindle angles measured in (A) mitotic and (B) meiotic germ cells of 15-, 20- and 30-day-old rats were grouped into three different categories $0-30^{\circ}$ (white bars), $30-60^{\circ}$ (grey bars) and $60-90^{\circ}$ (black bars), and they were plotted as the proportion of cells in each range. (A) Mitotic cells show a proportion of cells in the range of $60-90^{\circ}$ (perpendicular) significantly higher than the other categories at the studied ages. (B) Meiotic cells did not show a clear preference for any particular range at any of the studied ages. Different letters indicate a significant difference $(P<0.05)$. Frequency of different spindle angles measured in (C) mitotic and (D) meiotic germ cells of the three studied ages. (C) The graph shows that the mitotic germ cells have a preference for angles around $80^{\circ}$, contrary to meiotic germ cells where they showed a slight preference for angles around $55^{\circ}$.

20-day-old rats, the rats were killed after $2 \mathrm{~h}$ and the orientation of the mitotic spindles was analysed in mitotically dividing cells. We did not find any major alterations in the histological organization of the testes. Metaphase plates were readily detected and the chromosomes were still aligned, suggesting that the drug did not disrupt the spindle microtubules (Fig. 5A). The mean angle of spindles in Nocodazole-treated cells was significantly smaller than in controls (Fig. 5B; $P<0.05)$. Next, we analysed whether Nocodazole treatment had a different effect upon mitotic or meiotic cells. Results showed that the proportion of meiotic cells in the ranges of $0-30^{\circ}, 30-60^{\circ}$ and $60-90^{\circ}$ showed no significant difference between control (white bars) and Nocodazole treatment (Fig. 5C, black bars). On the contrary, the proportion of mitotic spindles in the range of $0-30^{\circ}$ was significantly higher than the percentage of spindles in the range of $60-90^{\circ}$ in Nocodazole-treated testes (Fig. 5D, white bars; $P<0.05)$. This result is contrary to that observed in controls, where the proportion of spindles in the range of $60-90^{\circ}$ was significantly higher than that in the range of $0-30^{\circ}$ (Fig. 5D, black bars; $P<0.05$ ). In addition, the proportion of mitotic spindles in the range of $0-30^{\circ}$ was significantly higher in Nocodazole-treated testes than in control testes (Fig. 5D, compare white with black bars; $P<0.05)$. A more in-depth analysis of
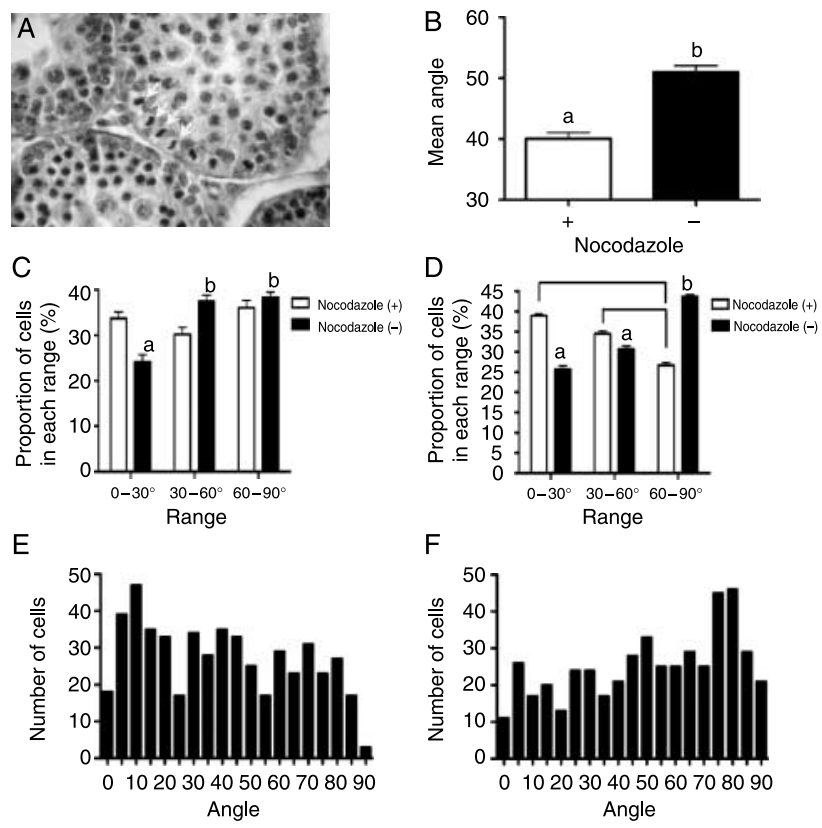

Figure 5 Nocodazole reduces the spindle angle of germ cells in 20-dayold rats. (A) Nocodazole $40 \mathrm{nM}$ did not change the histology of seminiferous tubules or chromosome alignment of the mitotic metaphase plates (arrows). (B) The average angle of the spindle in Nocodazole-treated testes (white bar) was significantly lower than control (black bar; $P<0.05$ ). (C and D) Distribution of the percentages of meiotic and mitotic spindles in three different ranges in both control (black bars) and Nocodazole-treated testes (white bars). Asterisks and different letters indicate significant differences $(P<0.05)$. (E) Frequency of different angles of mitotic spindles after Nocodazole treatment or $(\mathrm{F})$ in control animals. The data were obtained from 600 measurements of three different rats.

the frequency of angles showed that the highest frequency of mitotic spindles in Nocodazole-treated testes was in the range of $5-10^{\circ}$ (Fig. 5E), whereas spindles of control cells were found mainly in the range of $75-80^{\circ}$ (Fig. 5F).

Thus, our results strongly suggest that pulling forces, probably mediated by astral microtubules, may be involved in positioning the mitotic spindle, but not the meiotic spindle.

\section{Discussion}

In this work, we have shown that mitotic spindles of spermatogonia are mainly perpendicularly oriented to the basement membrane of the seminiferous tubules, unlike those cells undergoing meiosis, which do not show any particular preference of spindle angle (perpendicular or parallel in relation to the basement membrane). In addition, we showed that the mechanism used by spermatogonia to undergo OCD probably involves astral microtubules pulling forces. Thus, our results suggest unanticipated sharp differences between mitotic and meiotic cell divisions in the mammalian testes. 
The pubertal establishment and adult maintenance of spermatogenesis require precursor cells not to be positioned asymmetrically within the tubule but rather uniformly. Transplantation of a single spermatogonium will result initially in asymmetrical spermatogenesis, but shortly thereafter in uniform spermatogenesis alongside the internal border of the entire tubule (Parreira et al. 1998). Thus, spermatogonial stem cells are capable of movement. Spermatogenesis is established at the speed of $\sim 60 \mu \mathrm{m} /$ day along the length of the seminiferous tubule after transplantation into an infertile recipient (Nagano et al. 1999). It has been suggested that the A-differentiating (A1-A4) population of spermatogonia can spread and populate the tubule (Chiarini-Garcia et al. 2003, Yoshida et al. 2007). The spermatogonia population, as well as the other populations, can be identified in the adult testis based upon morphological criteria, in addition to localization in specific stages of the cycle. Some molecular markers like c-kit, Notch, Sox3 and neurogenin3 have been identified in spermatogonia, but they cannot discriminate among the different cell types (e.g. A1 from A2; Mori et al. 2003, von Schonfeldt et al. 2004, Raverot et al. 2005). Another criterion has been the use of flow cytometry and germ cell transplantation, and a spermatogonia population displaying $\alpha_{v}$-integrin ${ }^{-/ d i m} \alpha_{6}$-integrin ${ }^{+}$Thy- $1^{\text {lo/+ }} \mathrm{C}^{-k i t^{-}}$ cells (Kubota et al. 2003, Ohbo et al. 2003). However, our colleagues were not able to distinguish different spermatogonia subtypes $\left(A_{a l}\right.$ from $\left.A_{S}\right)$. This situation becomes more complicated in the case of mitotic cells where no nuclear morphology can be used to determine the specific cell type. Hence, we were not able to discriminate the spindle behaviour between the different spermatogonia populations in this work. Perhaps, future studies could focus on adult animals to try and distinguish the spindle behaviour of different spermatogonia populations. The capability of stem cells to move and the uniform organization of spermatogenesis alongside the internal border of seminiferous tubules suggest that some regulatory influence must govern the even distribution of spermatogenic cells during normal spermatogenesis. In this study, we observed that around $60 \%$ of the mitotically dividing germ cells showed spindle angles smaller than $60^{\circ}$, which represents a parallel or randomly distributed orientation. These cells could represent the moving spermatogonia population spreading laterally to occupy the entire border of the seminiferous tubules. In fact, it has been suggested that the differentiating spermatogonia (A1-A4 cells, intermediate type and type B spermatogonia) move laterally on the seminiferous tubule plane. These occur progressively as the more differentiated cells divide and are pushed laterally from the interstitial location (Chiarini-Garcia et al. 2001). In fact, recent data have shown that A-undifferentiated spermatogonia have a biased localization to the vascular network and accompanying Leydig cells (Yoshida et al. 2007). As these cells undergo mitosis, they begin to scatter and colonize the inner surface of the seminiferous tubules. These data are compatible with the hypothesis that A-undifferentiated spermatogonial cells may present spindles perpendicularly oriented to the basement membranes of the seminiferous tubules. On the other hand, the differentiating spermatogonia (A1-A4) could be the moving cell population exhibiting parallel or randomly oriented spindles. The OCD in the A-undifferentiated spermatogonia might be maintained by specific environmental signals, such as those described in the generation of goniablast cells during Drosophila melanogaster spermatogenesis, where the germ stem cell undergoes mitosis and the mother cell remains attached to the hub cell, due to the perpendicular orientation of the spindle relative to the basement membrane (Watt \& Hogan 2000, Li \& Xie 2005, Kirilly \& Xie 2007, Yamashita et al. 2007). In this work, we have shown that the mitotic plane orientation of basal located spermatogonia is similar to that observed in Drosophila and C. elegans, indicating a phylogenetically conserved feature in germ stem cells. These data suggest that the intrinsic and environmental determinants involved in maintaining the 'stemness' of germ stem cells may be conserved between these species. In Drosophila, it has been shown that the cytokine-like ligand unpaired (Upd) expressed by hub cells activates the Janus kinase-signal transducers and controls the self-renewal of germ stem cells. Mammalian spermatogonia are in contact with the basement membrane and with their Sertoli cells, but no Hub cell alike has been described in this system. Thus, paracrine signals controlling the stem cell compartment and spindle orientation must come from either the Sertoli cells or the basement membrane. In this context, the Sertoli cells produce and probably secrete proteins that could fulfil these functions, such as GDF (growth differentiation factor) or Jagged-1, which may also act to maintain the germ cell compartment (Mori et al. 2003, von Schonfeldt et al. 2004). The biochemical and molecular nature of the physical site where stem cells are located is still unknown but some studies have shown that the extracellular matrix might guide the orientation of the division axis in an in vitro culture of HeLa cells (Thery et al. 2005). This is consistent with the ability of laminin, a major basement membrane component of the seminiferous tubule, to support spermatogonial stem cell growth (Hamra et al. 2004).

Contrary to spermatogonia, meiotically dividing germ cells may not require specific environmental signals to divide, since their progeny only gives rise to two similar cells. In addition, these cells do not need to migrate laterally as spermatogonial germ cells do; their spindles do not have a preference for any particular orientation. A growing population of basal cells allows the meiotic cells to advance towards the lumen of the seminiferous tubules. It was interesting to see that the mean spindle angle varies and appears to have small, but significant 
fluctuations between the studied ages. Similar results were found in the developing telencephalon where the mitotic spindles rotate according to embryonic age (Haydar et al. 2003). In our system, the spindle measurements from 5-day-old rats may reflect the mitosis of both germ and Sertoli cells. In the rat, Sertoli cells begin to divide early after birth and they continue to increase their number up to around the second week after birth (Cupp \& Skinner 2005). They then differentiate and only germ cells continue in the proliferative state (Petersen \& Soder 2006). Thus, our determinations of the spindle orientation in 5- and 10-day-old rats may be 'contaminated' with spindles of Sertoli cells, which cannot be distinguished from germ cell spindles. However, our determinations in later days of development (15-30 days) correspond only to germ cells and cannot be attributed to Sertoli cells.

Experiments done in one-cell-stage $C$. elegans embryos have shown that astral microtubule pulling forces are pervasive throughout the cell cortex, and that these pulling forces are stronger on one side of the cell, at the posterior cortex, causing the spindle to shift from the centre of the embryo towards the posterior (Labbe et al. 2004). Here, we have shown that low amounts of Nocodazole ( $40 \mathrm{nM})$, a drug that depolymerizes microtubules, are able to change the orientation angle of dividing mitotic germ cells. Nocodazole has been widely used to depolymerize cell microtubules in both interphasic cells and spindle microtubules in mitotic cells (Moreno et al. 2006); however, low amounts of this drug (20-40 nM) depolymerize only astral and not spindle microtubules (O'Connell \& Wang 2000). The procedure of intratesticular injection to administer a drug targeting germ cells has been previously used to deliver a cellpermeable caspase-2 inhibitor in pubertal male rats and also to deliver an apoptosome inhibitor (minocycline) in adult mice. Both groups demonstrated that the drugs reach all intratubular cells (Castanares et al. 2005, Zheng et al. 2006). Even though we were not able to demonstrate the presence of a bona fide spindle, we never observed disabled metaphase plates, suggesting that chromosomes were kept aligned at the cell's centre by spindle microtubules. In fact, a higher concentration of Nocodazole $(10 \mu \mathrm{M})$ induced a disorganization of the metaphase plates in the rat testes (data not shown). Therefore, we propose that pulling forces from astral microtubules might be necessary to achieve OCDs in germ cells. It was interesting to see that these pulling forces seem to be more relevant to keep the perpendicular orientation of the spindles in spermatogonia than in spermatocytes. These results may be related to the fact that the spindle of a meiotic germ cell is randomly positioned and the astral microtubules exert a similar force on both sides of the spindle. On the other hand, the spindles of spermatogonia have to rotate in order to be aligned with the perpendicular plane of the cell. After Nocodazole treatment, this asymmetric pulling force, probably from astral microtubules, disappears and the spindle moves to a possible resting state, relatively parallel to the basement membrane of the seminiferous tubule.

For the first time, we have clearly shown a difference in the spindle angle of division between mitotic and meiotic germ cells in rat spermatogenesis. These results were not anticipated and they provide new evidence to help understand the mechanisms involved in the development and maintenance of germ stem cells and the differences between mitotic and meiotic spindles in the same developmental process of differentiation.

\section{Materials and Methods}

\section{Animals}

Male Sprague-Dawley rats between the ages of 5 and 30 days, three animals of each age, were acquired from the Animal Facility of our Faculty. The rats were housed under a 12L:12D cycle with water and rat chow ad libitum and were killed by cervical dislocation. Investigations were conducted in accordance with the rules laid down by the Consortium for Developing a Guide for the Care and Use of Agricultural Animals in Agricultural Research and Teaching and by the National Research Council. All animal protocols were endorsed by the Chilean National Fund of Science and Technology (FONDECYT).

\section{Intratesticular injections}

Twenty-day-old rats were anaesthetized with ketamine: xylazine (1 mg/kg: $750 \mathrm{mg} / \mathrm{kg}$ ) i.m. The testes were exteriorized through a low midline incision. Ten microlitres of a solution containing $40 \mathrm{nM}$ Nocodazole was infused via a $30 \mathrm{G}$ needle inserted through the tunica albuginae with the tip resting in the testicular interstitium. Following drug delivery, the testes were returned to the peritoneum and the incision was closed. The rats were killed $2 \mathrm{~h}$ after injection for the assessment of germ cell spindle orientation. As a control, DMSO $(1 \mu \mathrm{M}$ final concentration) was injected in the testes. Three different rats were used for all experiments.

\section{Testes histology}

Rat testes of different ages were fixed in Bouin solution (15 parts of picric acid-aqueous solution, 5 parts of formaldehyde and 1 part of glacial acetic acid). Tissues were fixed at least overnight at room temperature. Then, they were embedded in paraffin, sectioned in sheets of $5 \mu \mathrm{m}$ and mounted on xylanized slides. Histological sections were deparaffinized through a xylol series, hydrated through an alcohol series and then washed with water. Slides were stained with hematoxylin/eosin, according to standard procedures. Slides were observed under an Olympus BH-2 microscope (Olympus, Tokyo, 
Japan). Pictures were acquired by a digital Nikon photo camera model CoolPix 4500 (Nikon, Tokyo, Japan).

\section{Spindle angle determination}

The spindle orientation in dividing rat germ cells was determined by first calculating the angle between an imaginary line drawn parallel to the metaphase plate of the cell and the tangent line aligned with the basement membrane of the seminiferous tubules (angle X, Fig. 3). The spindle angle was calculated as the difference between angle $X$ and $90^{\circ}$. Using this procedure, we obtained the angle $\alpha$ representing the orientation of the spindle in relation to the basement membrane.

In order to study the mitotic or meiotic germ cell spindle, we took advantage of the differential position of these cells in the seminiferous tubules. Mitotic spindles dividing spermatogonia are always attached to the basement membrane, and they are easily identified by optical microscopy (Fig. 3A). On the other hand, meiotically dividing spermatocytes are always located in the second or third cell layer towards the lumen of the seminiferous epithelium (Fig. 3B; Clermont 1972). Thus, mitotically and meiotically dividing germ cells are clearly identified in developing rat testes. All determinations were performed by two different observers in three different rats, and 200 spindles were measured in each (a total of 600 spindles per determination). Data acquisition was done with Image Tool 3.0 from San Antonio Dental School, Univ Texas (San Antonio, TX, USA): data are shown as the mean angle \pm S.E.M. or the frequency of each angle at any developmental age of the rat.

\section{Statistical analysis}

Kruskal-Wallis test was used in experiments with multiple groups, and Mann-Whitney test to compare with groups. Statistical significance was defined as $P<0.05$ (Sokal 1995). We measured 200 spindles in three animals; therefore, data were calculated with a total of 600 measurements of each experimental point.

\section{Acknowledgements}

Part of this work was financed by a grant from the Chilean Research Council (FONDECYT, 1040800 and 1070360) to R D M. We thank Mr Jurriaan Brouwer-Visser for his excellent assistance with English grammar. Grant Sponsor: National Commission for Scientific Research and Technology (FONDECYT 1070360). The authors declare that there is no conflict of interest that would prejudice the impartiality of this scientific work.

\section{References}

Adams RJ 1996 Metaphase spindles rotate in the neuroepithelium of rat cerebral cortex. Journal of Neuroscience 16 7610-7618.

Baena-Lopez LA, Baonza A \& Garcia-Bellido A 2005 The orientation of cell divisions determines the shape of Drosophila organs. Current Biology 15 $1640-1644$.
Bellaiche Y \& Gotta M 2005 Heterotrimeric G proteins and regulation of size asymmetry during cell division. Current Opinion in Cell Biology $\mathbf{1 7}$ 658-663.

Billig H, Furuta I, Rivier C, Tapanainen J, Parvinen M \& Hsueh AJ 1995 Apoptosis in testis germ cells: developmental changes in gonadotropin dependence and localization to selective tubule stages. Endocrinology 136 5-12.

Castanares M, Vera Y, Erkkila K, Kyttanen S, Lue Y, Dunkel L, Wang C, Swerdloff RS \& Sinha Hikim AP 2005 Minocycline up-regulates BCL-2 levels in mitochondria and attenuates male germ cell apoptosis. Biochemical and Biophysical Research Communications 337 663-669.

Chiarini-Garcia H, Hornick JR, Griswold MD \& Russell LD 2001 Distribution of type A spermatogonia in the mouse is not random. Biology of Reproduction 65 1179-1185.

Chiarini-Garcia H, Raymer AM \& Russell LD 2003 Non-random distribution of spermatogonia in rats: evidence of niches in the seminiferous tubules. Reproduction 126 669-680.

Clermont Y 1972 Kinetics of spermatogenesis in mammals: seminiferous epithelium cycle and spermatogonial renewal. Physiological Reviews $\mathbf{5 2}$ 198-236.

Cupp AS \& Skinner MK 2005 Embryonic Sertoli cell differentiation. In Sertoli Cell Biology, pp 43-91. Eds MK Skinner \& MD Griswold. Elsevier: Pullman.

Geldmacher-Voss B, Reugels AM, Pauls S \& Campos-Ortega JA 2003 A 90-degree rotation of the mitotic spindle changes the orientation of mitoses of zebrafish neuroepithelial cells. Development 130 3767-3780.

Hamra FK, Schultz N, Chapman KM, Grellhesl DM, Cronkhite JT, Hammer RE \& Garbers DL 2004 Defining the spermatogonial stem cell. Developmental Biology 269 393-410.

Haydar TF, Ang E Jr \& Rakic P 2003 Mitotic spindle rotation and mode of cell division in the developing telencephalon. PNAS 100 2890-2895.

Kirilly D \& Xie T 2007 The Drosophila ovary: an active stem cell community. Cell Research 17 15-25.

Kubota H, Avarbock MR \& Brinster RL 2003 Spermatogonial stem cells share some, but not all, phenotypic and functional characteristics with other stem cells. PNAS 100 6487-6492.

Labbe JC, McCarthy EK \& Goldstein B 2004 The forces that position a mitotic spindle asymmetrically are tethered until after the time of spindle assembly. Journal of Cell Biology 167 245-256.

Lechler T \& Fuchs E 2005 Asymmetric cell divisions promote stratification and differentiation of mammalian skin. Nature 437 275-280.

Li L \& Xie T 2005 Stem cell niche: structure and function. Annual Review of Cell and Developmental Biology 21 605-631.

Malkov M, Fisher Y \& Don J 1998 Developmental schedule of the postnatal rat testis determined by flow cytometry. Biology of Reproduction $\mathbf{5 9}$ 84-92.

McCarthy EK \& Goldstein B 2006 Asymmetric spindle positioning. Current Opinion in Cell Biology 18 79-85.

Moreno RD, Palomino J \& Schatten G 2006 Assembly of spermatid acrosome depends on microtubule organization during mammalian spermiogenesis. Developmental Biology 293 218-227.

Mori S, Kadokawa Y, Hoshinaga K \& Marunouchi T 2003 Sequential activation of Notch family receptors during mouse spermatogenesis. Development, Growth and Differentiation 45 7-13.

Na J \& Zernicka-Goetz M 2006 Asymmetric positioning and organization of the meiotic spindle of mouse oocytes requires CDC42 function. Current Biology 16 1249-1254.

Nagano M, Avarbock MR \& Brinster RL 1999 Pattern and kinetics of mouse donor spermatogonial stem cell colonization in recipient testes. Biology of Reproduction 60 1429-1436.

O'Connell CB \& Wang YL 2000 Mammalian spindle orientation and position respond to changes in cell shape in a dynein-dependent fashion. Molecular Biology of the Cell 11 1765-1774.

Ohbo K, Yoshida S, Ohmura M, Ohneda O, Ogawa T, Tsuchiya H, Kuwana T, Kehler J, Abe K, Scholer HR \& Suda T 2003 Identification and characterization of stem cells in prepubertal spermatogenesis in mice small star, filled. Developmental Biology 258 209-225.

Parreira GG, Ogawa T, Avarbock MR, Franca LR, Brinster RL \& Russell LD 1998 Development of germ cell transplants in mice. Biology of Reproduction 59 1360-1370. 
Petersen C \& Soder O 2006 The sertoli cell - a hormonal target and 'super' nurse for germ cells that determines testicular size. Hormone Research 66 153-161.

Raverot G, Weiss J, Park SY, Hurley L \& Jameson JL 2005 Sox3 expression in undifferentiated spermatogonia is required for the progression of spermatogenesis. Developmental Biology 283 215-225.

de Rooij DG \& Russell LD 2000 All you wanted to know about spermatogonia but were afraid to ask. Journal of Andrology 21 776-798.

Russell L, Ettlin R, Hikim A \& Clegg E 1990 Histological and Histopathological Evaluation of the Testis, Clearwater: Cache River Press, p 474.

von Schonfeldt V, Wistuba J \& Schlatt S 2004 Notch-1, c-kit and GFRalpha1 are developmentally regulated markers for premeiotic germ cells. Cytogenetic and Genome Research 105 235-239.

Sokal RR 1995 Biometry: The Principles and Practice of Statistic in Biological Research, New York: W.H. Freeman, p 887.

Thery M, Racine V, Pepin A, Piel M, Chen Y, Sibarita JB \& Bornens M 2005 The extracellular matrix guides the orientation of the cell division axis. Nature Cell Biology 7 947-953.

Tibber MS, Kralj-Hans I, Savage J, Mobbs PG \& Jeffery G 2004 The orientation and dynamics of cell division within the plane of the developing vertebrate retina. European Journal of Neuroscience 19 497-504.
Watt FM \& Hogan BL 2000 Out of Eden: stem cells and their niches. Science 287 1427-1430.

Yamashita YM, Mahowald AP, Perlin JR \& Fuller MT 2007 Asymmetric inheritance of mother versus daughter centrosome in stem cell division. Science 315 518-521.

Yoshida S, Sukeno M \& Nabeshima Y 2007 A vasculature-associated niche for undifferentiated spermatogonia in the mouse testis. Science $\mathbf{3 1 7}$ 1722-1726.

Zheng S, Turner TT \& Lysiak JJ 2006 Caspase 2 activity contributes to the initial wave of germ cell apoptosis during the first round of spermatogenesis. Biology of Reproduction 74 1026-1033.

Received 27 August 2007

First decision 1 October 2007

Revised manuscript received 4 January 2008

Accepted 22 January 2008 